\title{
In-Hospital Outcomes of Inflammatory Bowel Diseases in Patients With Diabetes Mellitus: A Propensity Score Matching Analysis
}

Ese Uwagbale ${ }^{1}$, Omolara G. Adeniran ${ }^{1}$, Olayemi A. Adeniran ${ }^{2,}$, , Ifeanyichukwu Onukogu ${ }^{1}$, Solomon Agbroko $^{3}$, Niket Sonpal ${ }^{4}$

1. Internal Medicine, Brookdale University Hospital Medical Center, Brooklyn, USA 2. Epidemiology, West Virginia University, Morgantown, USA 3. Obstetrics and Gynecology, Maimonides Medical Center, Brooklyn, USA 4. Gastroenterology and Hepatology, Brookdale University Hospital Medical Center, Brooklyn, USA

Corresponding author: Ese Uwagbale, eseuwagbale@gmail.com

\section{Abstract \\ Introduction}

Inflammatory bowel disease (IBD) is increasingly common among patients with other comorbid chronic conditions, particularly diabetes mellitus (DM). Yet, studies that explored the impact of comorbid diabetes on the outcomes of IBD are scanty. Therefore, this study aims to examine the outcomes of inflammatory bowel disease among hospitalized patients with diabetes mellitus.

\section{Methods}

Using the Nationwide Inpatient Sampling (NIS) database from 2016 to 2018, we identified patients' records with a diagnosis of IBD using the International Classification of Diseases, Tenth Revision codes (ICD-10). The overall study population was further stratified by diabetes mellitus status. We matched patients with IBD and diabetes mellitus (IBD DM) with IBD cohorts using a greedy propensity score matching (PSM) ratio of 1:1 and compared in-hospital outcomes between the two cohorts. Conditional logistic regression was performed to estimate the odds of outcomes.

\section{Results}

Out of the 192,456 hospitalizations for IBD, 34,073 (7.7\%) had comorbid IBD DM and 158,383 (92.3\%) had no diabetes mellitus (IBD only). Patients with IBD DM are likely to be older. They have higher rates of hypertension, hyperlipidemia, coronary artery disease, obesity, peripheral vascular disease, congestive heart failure, chronic kidney disease, chronic lung disease, chronic liver disease, and stroke than the IBD cohort. After propensity score matching, IBD DM was associated with a lower adverse outcome [odds ratio (OR): 0.96, confidence interval (CI): 0.93 - 0.99, p < 0.01], IBD-related complications (intestinal or rectal fistula, intra-abdominal abscess, toxic colitis, intestinal perforation, intestinal obstruction, toxic megacolon, abscess of the abdomen, and perianal abscess), (OR: 0.76, CI: $0.72-0.80, \mathrm{P}<0.01$ ), IBD-related surgery (intestinal resections, incision, and excisions of intestine and manipulations of the rectosigmoid, rectal and

Review began 06/14/2021 Review ended 07/08/2021 Published 07/22/2021

\section{() Copyright 2021}

Uwagbale et al. This is an open access article distributed under the terms of the Creative Commons Attribution License CC-BY 4.0., which permits unrestricted use, distribution, and reproduction in any medium, provided the original author and source are credited. perianal) (OR: 0.90, CI: 0.85 - 0.95, P <0.01). Furthermore, IBD DM was associated with a higher sepsis complication than the IBD-only cohort (OR: 1.24, CI: $1.19-1.30, \mathrm{P}<0.01$ ).

\section{Conclusion}

Our results highlight the extent to which diabetes mellitus impacts IBD outcomes and prognosis. Additionally, they emphasize the clinical awareness needed in the management of those with comorbid diseases.

Categories: Internal Medicine, Gastroenterology, Public Health

Keywords: inflammatory bowel disease, diabetes mellitus, adverse clinical outcomes, inflammatory bowel disease association with diabetes, complications of inflammatory bowel disease

\section{Introduction}

Inflammatory bowel disease (IBD) is a chronic non-infectious inflammation of the gastrointestinal tract, associated with significant morbidities and poor quality of life [1-3]. IBD comprises Crohn's disease and ulcerative colitis, which constitute a significant clinical and public health burden affecting about 7-million people worldwide [4]. The United States contributes a quarter of the total global patients with IBD with an age-standardized prevalence rate of 464.5 per 100,000 population in 2017 [4]. IBD results from a complex interaction of the genetic and environmental factors that disrupt the immune mechanisms of the gastrointestinal tract and result in inflammation [5-6]. While IBD affects mainly the gastrointestinal tract, the associated chronic systemic inflammation leads to several extraintestinal manifestations affecting the body's major organs [7-8]. In addition, with the rise in the aging population living with IBD, there is an 
increased likelihood of being affected with other comorbid conditions, including cardiovascular disease or diabetes mellitus (DM) [9]. These concomitant chronic diseases modify the progression of IBD and might lead to long periods of intermittent relapses and exacerbations [10].

DM is one of the most common comorbid conditions in the United States. DM is a chronic metabolic disorder characterized by persistent impaired blood glucose metabolism [11]. According to the National Diabetes Statistics Report, 34.2-million people currently live with diabetes in the United States [12]. As a chronic disease that affects most body organs, DM can modify disease outcomes and lead to other complications. Published studies have reported IBD DM to have a higher incidence of complications, including exacerbations requiring prolonged hospitalizations and the need for surgical interventions [10]. Also, prior studies have reported DM as a covariate associated with increased risk of hospitalizations, infectious complications, colorectal cancers, and mortality [13-14]. However, studies that describe the outcomes of IBD among diabetic patients are minimal. Understanding the hospital outcomes of comorbid IBD DM is vital for improving management and reducing morbidity and mortality. Therefore, this study sought to investigate the role of DM on IBD using a national hospital database.

\section{Materials And Methods}

\section{Study data}

This study utilized data from the Nationwide Inpatient Sample (NIS) 2016-2018 to perform a retrospective cohort study. The NIS is the largest hospital database in the United States, containing discharge records of about 8-million hospital stays annually. The NIS is a stratified, clustered database that samples discharge records from 20 percent of non-federal community hospitals. Each discharge record contains diagnoses and procedures coded using the International Classification of Disease, 10th revision (ICD-10). Institutional Review Board approval was not required for this study since the data has been de-identified.

\section{Study population}

The study population consisted of adults aged 18 and older with a diagnosis of IBD from January 1, 2016, to December 31, 2018, identified using the ICD 10: K50-K51. After excluding patients with missing age, mortality, and sex variables, we categorized the total study population into two groups: patients with IBD DM and those with IBD only. We defined DM status using ICD-10. Comparative analyses were conducted between the two groups regarding demographics such as age, sex (male and female), race/ethnicity (Whites, Blacks, Hispanics, and other races), income status (categorized into four according to the average household income of the zip-code), hospital bed size (small, medium, and large hospitals), and comorbidities (hypertension, hyperlipidemia, coronary artery disease, obesity, peripheral vascular disease, chronic heart failure, chronic kidney disease, chronic lung disease, chronic liver disease, stroke).

\section{Study outcomes}

The outcomes of this study were in-hospital adverse events, a composite of in-hospital mortality, IBDrelated complications (development of fistula, abscess, colitis, perforation, intestinal obstruction, toxic megacolon), intestinal surgery (intestinal resections, incision, and excisions of intestine and manipulations of the rectosigmoid, rectal and perianal), sepsis, and septicemia, clostridium difficile infections, colorectal cancer, and resources utilization measures (length of stay and cost of hospitalizations).

\section{Statistical analysis}

Weighted values are generated to obtain a nationally representative estimate of the hospitalized patients and then produce median values and percentages for the variables. Continuous variables were expressed as weighted median values with interquartile range and compared between the cohorts using independent $\mathrm{t}$ tests. In contrast, categorical variables were expressed as percentages and compared using the chi-square test. Patient demographics, comorbidities, hospital characteristics, and in-hospital outcomes were compared between males and females. We used the cost-to-charge ratio files provided by the Healthcare Cost and Utilization Project (HCUP) to convert the hospital charges to more accurate hospital costs for cost calculation. A p-value of $<0.05$ was considered statistically significant.

A propensity matching method was implemented to derive two cohorts of matched samples to control potential confounding factors. A propensity score was derived for each observation via a multivariate logistic regression that models the odds of DM and the baseline characteristics. A nearest-neighbor with a ratio of $1: 1$, balanced propensity matching was made using a caliper width cut-off $<0.2$ of the standard deviation of the propensity score. A p-value of $<0.05$ was considered statistically significant. After outcomes were compared between propensity score-matched subjects (IBD subjects with and without DM). The paired t-test was used to compare continuous variables and the McNemar test for categorical variables between the matched cohorts.

Finally, we developed many conditional generalized logistic regression models accounting for the matching pair, with diabetes as a primary predictor. Each of the outcomes is the dependent variable in the models. The negative binomial model was used for continuous outcomes for the length of stay and gamma distributions 


\section{Cureus}

mode for cost. Data manipulation and statistical analyses were performed using SAS 9.4 software (SAS Institute Inc., Cary, NC). Propensity score matching (PSM) was performed using the Matchit package in $\mathrm{R}$ statistical software (version 3.5; R Foundation for Statistical Computing, Vienna, Austria).

\section{Results}

\section{Baseline characteristics of the study population}

From January 1, 2016, until December 31, 2018, a total of 21,400,282 hospitalizations were present in the NIS database. Of these hospitalizations, 192,456 had a diagnosis of IBD. DM was present in 7.7\% (34,073 records), as seen in Figure 1.

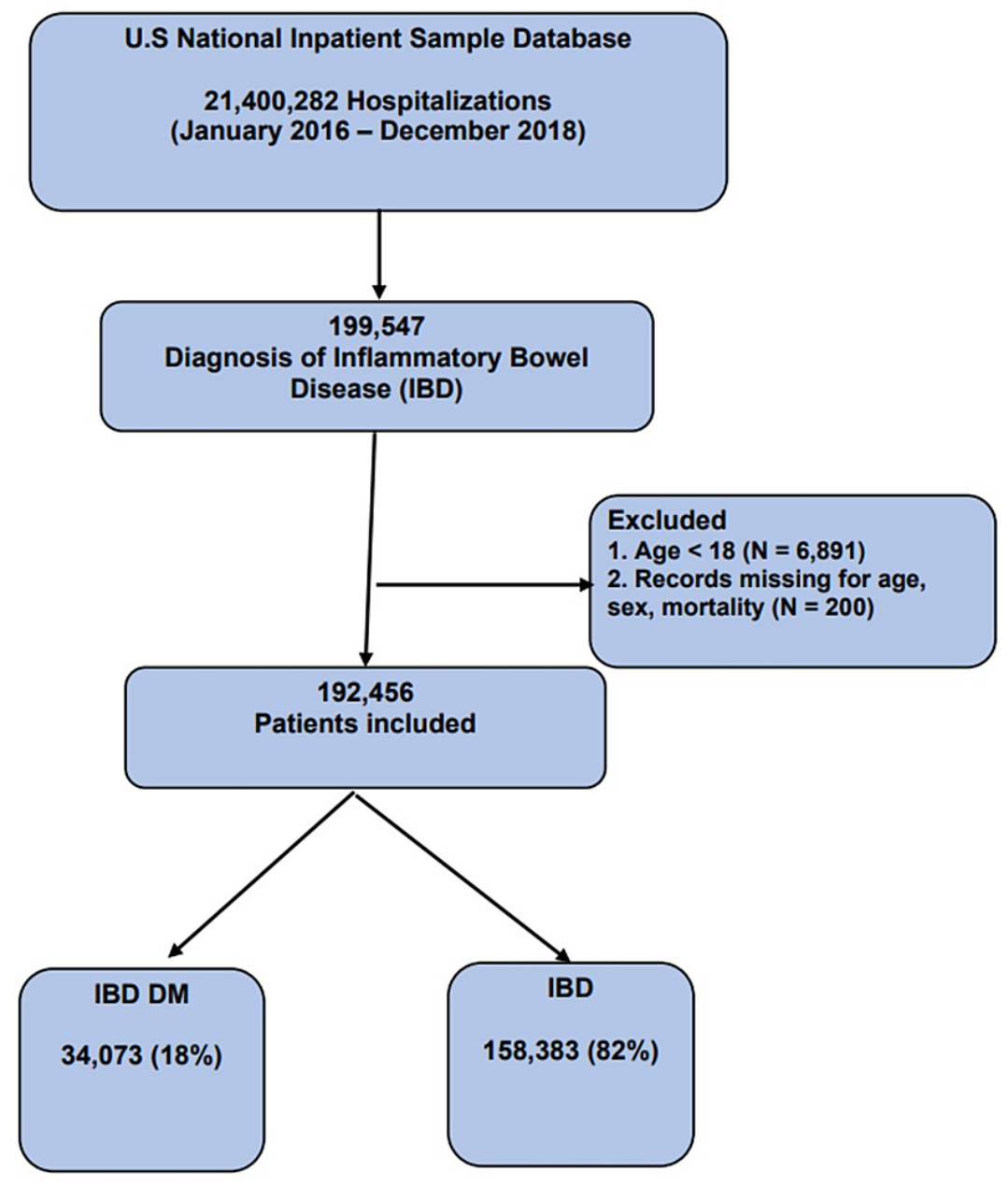

FIGURE 1: Baseline characteristics of the study population

There were significant differences in the baseline characteristics among the IBD DM and IBD cohorts. Compared to IBD patients, the IBD DM cohorts were older (median age 64.6 vs. 50.8), female (53.6\% vs. $46.4 \%$ ), and had a higher percentage of comorbidities than the IBD-only cohort. The baseline patient and hospital characteristics of the two groups are shown in Tables 1-2.

\begin{tabular}{|c|c|c|c|c|}
\hline \multirow[t]{3}{*}{ Variables } & Total & IBD DM & IBD & p-value \\
\hline & 192,456 & $(\mathrm{~N}=34,073)$ & $(\mathrm{N}=158,383)$ & \\
\hline & (WE: 962,280) & $(N=170,365)$ & $(\mathrm{N}=791,915)$ & \\
\hline Age (median, IQR) & $54.0(36.7-68.5)$ & $64.6(53.7-73.5)$ & $50.8(34.4-66.5)$ & $<0.0001$ \\
\hline
\end{tabular}




\section{Cureus}

Age category

$18-34$
$35-54$
$55-74$

3.8

24.3

29.3

21.7

30.9

50.9

$<0.0001$

$\geq 75$

15.8

23.6

30.6

14.1

Sex

Male

Female

Race

White $\quad 76.0$

Black

Hispanic

6.0

6.4

5.9

$<0.0001$

Other

Comorbidities

Hypertension

38.2

Hyperlipidemia

Coronary Artery Disease

Obesity

Peripheral Vascular Disease

Chronic Heart Failure

Chronic Kidney Disease

Chronic Lung Disease

Chronic Liver Disease

Stroke

Smoking

\section{Hospital Bed Size}

Small

Medium

28.1

Large

52.9

Median Household Income

$\begin{array}{lc}<25 \text { th percentile } & 27.2 \\ \text { 26-50th percentile } & 27.2 \\ 51-75 \text { th percentile } & 24.8 \\ 76-100 \text { th percentile } & 20.8\end{array}$

20.9

5.8

16.9

27.2
63.2

47.6

27.7

24.8

7.7

21.4

14.2

29.8

10.1

10.2

13.5
27.2

27.2

24.8

20.8

32.9

$<0.0001$

17.4

9.5

9.8

4.3

7.3

4.8

19.0

6.1

4.9

17.6

$<0.0001$

$<0.0001$

$<0.0001$

$<0.0001$

$<0.0001$

$<0.0001$

$<0.0001$

$<0.0001$

$<0.0001$

$<0.0001$

19.6

19.0

27.8

$<0.0001$

53.3

23.8

25.3

26.3

24.6 


\section{Cureus}

TABLE 1: Baseline characteristics of patients with inflammatory bowel disease and diabetes mellitus (Nationwide Inpatient Sampling database 2016 -2018)

Values are expressed in percentages unless otherwise stated.

WE: weighted estimates; IBD: inflammatory bowel disease; DM: diabetes mellitus; IQR: interquartile range

\begin{tabular}{|c|c|c|c|}
\hline Variables & IBD DM & IBD & SMD (\%) \\
\hline Observations & $(\mathrm{N}=33,870)$ & $(\mathrm{N}=33,870)$ & \\
\hline Age (median, IQR) & 64.5 (53.7 - 73.4) & 66.4 (54.6 - 76.0) & 10.3 \\
\hline Sex & & & 3.0 \\
\hline Male & 46.3 & 46.2 & \\
\hline Female & 53.7 & 53.8 & \\
\hline Race & & & 3.9 \\
\hline White & 74.7 & 78.7 & \\
\hline Black & 12.0 & 9.2 & \\
\hline Hispanic & 6.4 & 4.8 & \\
\hline Other & 3.9 & 3.3 & \\
\hline Unknown & 3.0 & 4.0 & \\
\hline \multicolumn{4}{|l|}{ Comorbidities } \\
\hline Hypertension & 63.0 & 65.6 & 5.5 \\
\hline Hyperlipidemia & 47.3 & 46.6 & 1.5 \\
\hline Coronary Artery Disease & 27.4 & 26.1 & 3.0 \\
\hline Obesity & 24.5 & 21.9 & 6.1 \\
\hline Peripheral Vascular Disease & 7.7 & 7.8 & 0.3 \\
\hline Chronic Heart Failure & 21.1 & 19.2 & 4.7 \\
\hline Chronic Kidney Disease & 14.0 & 12.3 & 4.9 \\
\hline Chronic Lung Disease & 29.7 & 30.2 & 1.1 \\
\hline Chronic Liver Disease & 10.1 & 9.8 & 1.0 \\
\hline Stroke & 10.2 & 10.1 & 0.4 \\
\hline Smoking & 13.6 & 13.7 & 0.3 \\
\hline Hospital Bed Size & & & 3.0 \\
\hline Small & 19.6 & 19.6 & \\
\hline Medium & 29.4 & 29.2 & \\
\hline Large & 51.0 & 51.2 & \\
\hline
\end{tabular}




\section{Cureus}

Median Household Income

$<25$ th percentile

26-50th percentile

51-75th percentile

76-100th percentile
27.1

27.2

24.9

20.8
26.5

27.0

25.6

20.8

TABLE 2: Baseline characteristics of patients with inflammatory bowel disease and diabetes mellitus, after propensity matching

Values are expressed in percentages unless otherwise stated.

SMD: standardized mean differences; IBD: inflammatory bowel disease; DM: diabetes mellitus; IQR: interquartile range

In-hospital outcomes are depicted in Table 3. Overall, the IBD DM cohorts had a lower prevalence of major in-hospital adverse events than the IBD cohorts. Compared to the IBD group, the IBD DM had lower IBDrelated complications $(9.3 \%$ vs. $14.5 \%, \mathrm{p}<0.01)$, intestinal surgeries $(26.0 \%$ vs. $38.5 \%, \mathrm{p}<0.01)$. On the other hand, the IBD DM group had a higher prevalence of sepsis and septicemia complications (14.7\% vs $10.8 \%$, $p$ $<0.01)$ and in-hospital mortality $(2.2 \%$ vs $1.4 \%, \mathrm{p}<0.01)$. The prevalence of clostridium difficile infections and colorectal cancer are similar between the two groups. Similarly, the length of hospital stay and costwere more prevalent among the IBD DM group than the IBD only group.

\begin{tabular}{|c|c|c|c|c|}
\hline In-hospital Outcomes & Total & IBD DM & IBD & $\mathrm{p}$-value \\
\hline Major Adverse events & $40.6 \%$ & $36.1 \%$ & $41.6 .1 \%$ & $<0.0001$ \\
\hline IBD-related complications & $13.5 \%$ & $9.3 \%$ & $14.5 \%$ & $<0.0001$ \\
\hline Surgery & $36.3 \%$ & $26.0 \%$ & $38.5 \%$ & $<0.0001$ \\
\hline Sepsis/Septicemia & $11.5 \%$ & $14.7 \%$ & $10.8 \%$ & $<0.0001$ \\
\hline C.difficile infections & $4.6 \%$ & $4.7 \%$ & $4.6 \%$ & 0.4205 \\
\hline Colorectal Cancer & $0.9 \%$ & $1.0 \%$ & $0.9 \%$ & 0.9339 \\
\hline Death & $1.5 \%$ & $2.2 \%$ & $1.4 \%$ & $<0.0001$ \\
\hline Length of stay (Median (IQR) & $3.1(1.7-3.7)$ & $3.5(1.9-6.3)$ & $3.0(1.7-5.5)$ & $<0.0001$ \\
\hline Cost (Median (IQR) & $8,224(4,986-14,761)$ & $9,224(5,582-16,212)$ & $8,036(4,873-14446)$ & $<0.0001$ \\
\hline
\end{tabular}

TABLE 3: In-hospital outcomes of inflammatory bowel disease among IBD DM vs IBD patients before propensity score matching

IBD: inflammatory bowel disease; DM: diabetes mellitus; IQR: interquartile range

Using propensity matching, we adjusted for baseline demographics and hospital characteristics to generate matched cohorts of IBD DM and IBD groups $(n=33,870)$ (Table 4). We achieved the variables' standardized mean differences between the two groups to arrive at a well-matched cohort. The overall in-hospital adverse events is still lower among the IBD DM cohort as compared to the IBD group ( $36.2 \%$ vs. $37.2 \%$, OR: 0.96 , CI: $0.93-0.99, \mathrm{p}<0.01)$. Furthermore, the IBD DM cohort had a lower incidence of IBD-related complication (9.3\% vs. $11.9 \%$, OR: 0.76, CI: $0.72-0.80$, P <0.01) and surgery (26.0\% vs. $28.9 \%$, OR: 0.90, CI: $0.85-0.95$, P $<.01)$. However, the DM group had a higher sepsis complication compared to the IBD group $(14.7 \%$ vs. $12.2 \%$, OR: 1.24 , CI: $1.19-1.30, \mathrm{P}<0.01)$. See Figures $2-4$ and Table 5. 


\section{Cureus}

\begin{tabular}{|c|c|c|c|c|}
\hline In-hospital Outcomes & IBD DM & IBD & OR (95\% CI) & p-value \\
\hline Major Adverse events & 36.2 & 37.2 & $0.96(0.93-0.99)$ & 0.0046 \\
\hline IBD-related complications & 9.3 & 11.9 & $0.76(0.72-0.80)$ & $<0.0001$ \\
\hline Surgery & 26.0 & 28.9 & $0.90(0.85-0.95)$ & 0.0004 \\
\hline Sepsis/Septicemia & 14.8 & 12.2 & $1.24(1.19-1.30)$ & $<0.0001$ \\
\hline C.difficile infections & 4.7 & 4.7 & $0.99(0.93-1.07)$ & $0.856 \%$ \\
\hline Colorectal Cancer & 1.0 & 1.0 & $0.96(0.82-1.12)$ & 0.5841 \\
\hline Death & 2.2 & 2.3 & $0.96(0.87-1.07)$ & 0.4670 \\
\hline Length of stay (Median IQR) & $3.5(1.9-6.3)$ & $3.3(1.8-6.1)$ & $1.03(1.02-1.05)$ & $<0.0001$ \\
\hline Cost (Median IQR) & $9,216(5,578-16,199)$ & $9,147(5,471-16,272)$ & $1.00(0.99-1.01)$ & 0.8839 \\
\hline
\end{tabular}

TABLE 4: In-hospital outcomes of inflammatory bowel disease among IBD DM vs IBD patients after propensity score matching

IBD: inflammatory bowel disease; DM: diabetes mellitus; IQR: interquartile range

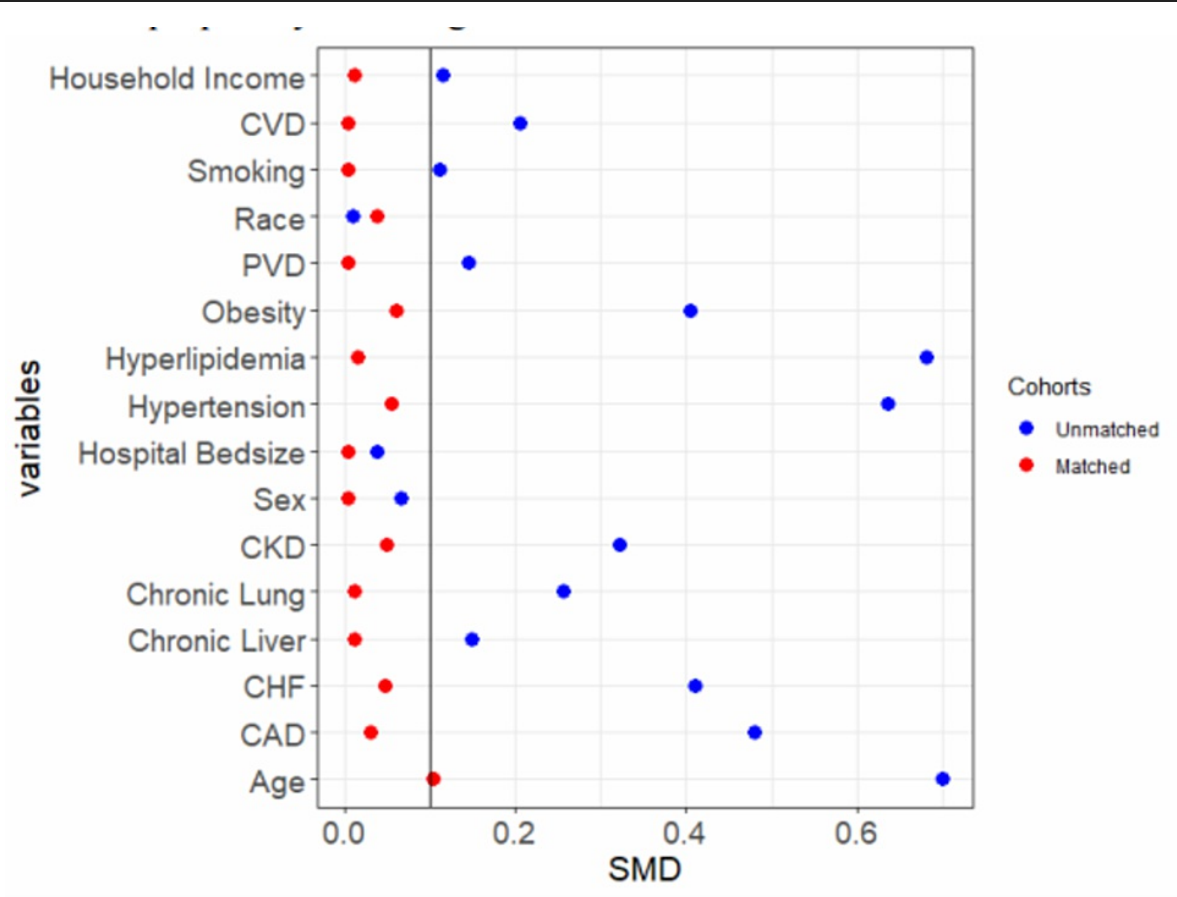

FIGURE 2: Dot plots showing the standardized mean differences of the baseline variables before and after propensity matching

CVD: cerebrovascular disease; PVD: peripheral vascular disease; CKD: chronic kidney disease; CHF: congestive heart failure; CAD: coronary artery disease 


\section{Cureus}

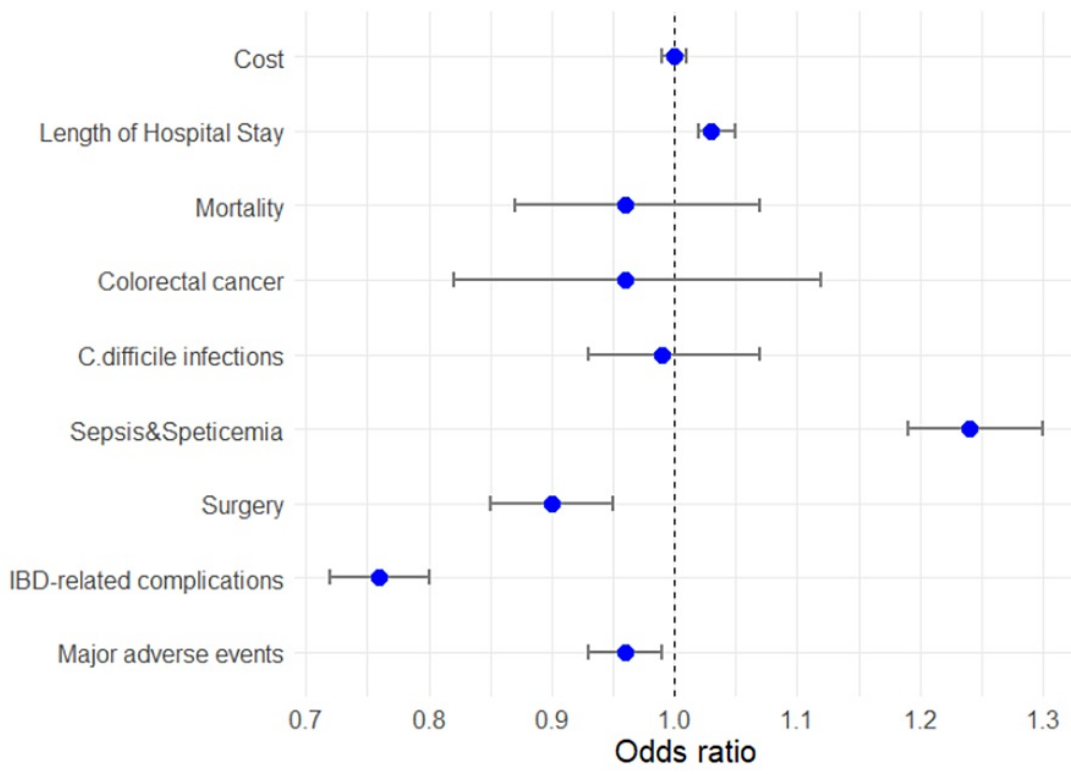

FIGURE 3: Plots of the adjusted odds ratio of clinical outcomes of inflammatory bowel disease and diabetes mellitus after propensity matching

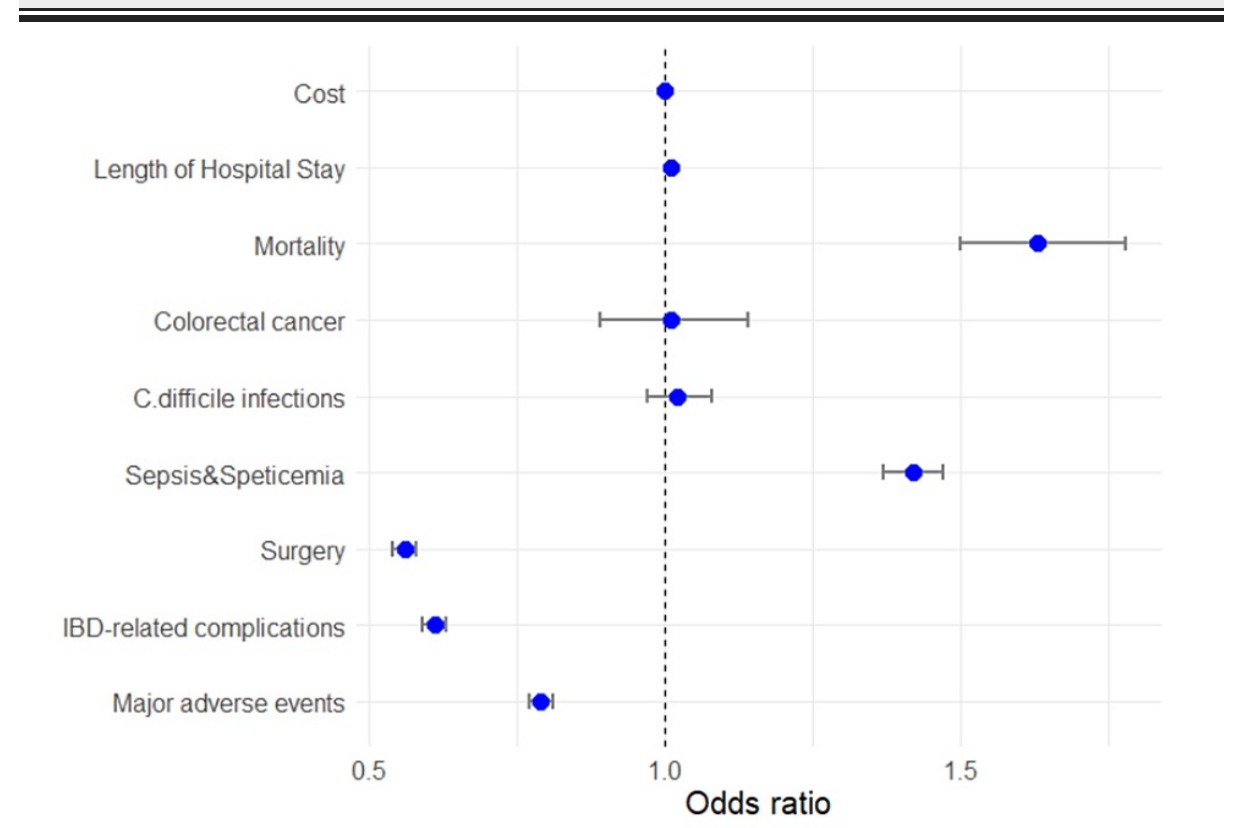

FIGURE 4: Plot of the unadjusted odds ratio of clinical outcomes of inflammatory bowel diseases and diabetes mellitus

\begin{tabular}{|c|c|c|c|}
\hline Variables & IBD DM & IBD & SMD (\%) \\
\hline Observations & $(\mathrm{N}=33,870)$ & $(\mathrm{N}=33,870)$ & \\
\hline Age (median, IQR) & $64.5(53.7$ - 73.4) & $66.4(54.6$ - 76.0) & 10.3 \\
\hline Sex & & & 3.0 \\
\hline
\end{tabular}




\section{Cureus}

Male $\quad 46.3$

White

Black

Other

Comorbidities

Hypertension 63.0

Hyperlipidemia

Coronary Artery Disease

Peripheral Vascular Disease

Chronic Heart Failure

Chronic Kidney Disease

Chronic Lung Disease

$<25$ th percentile
26-50th percentile
$51-75$ th percentile
$76-100$ th percentile

TABLE 5: Baseline characteristics of patients with inflammatory bowel disease and diabetes mellitus, after propensity matching

Values are expressed in percentages unless otherwise stated.

SMD: standardized mean differences; IBD: inflammatory bowel disease; DM: diabetes mellitus; IQR: interquartile range 


\section{Discussion}

In this study, we examined the in-hospital outcomes of IBD among patients with DM. In addition, we compared IBD patients with comorbid DM to those without diabetes using a propensity score matching method. The main findings of our study are as follows: (1) IBD DM patients have a significant increase in the odds of developing sepsis and septicemia compared to the IBD only cohort ; (2) IBD DM is associated with a decrease in the odds of having IBD-related complications and surgical interventions compared to IBD cohorts; (3) there were no significant differences in the risk of mortality, clostridium difficile infections, or colorectal cancer, among IBD DM and IBD.

To date, only a few clinical or epidemiological studies had investigated the IBD outcomes among diabetic patients. A recent longitudinal cohort study conducted in the US found that comorbid IBD DM patients have a significantly increased risk of IBD-related hospitalization, complications, surgical interventions, and allcause mortality [10]. Compared with IBD cohorts, IBD DM patients had significantly higher rates of sepsis and other infections [10]. Furthermore, a retrospective study that utilized 2810 outpatient cohorts concluded that DM is associated with worse IBD severity reflected by increased use of the emergency room and nearly double the rates of patients with gastrointestinal clinic visits [15]. While these studies have employed different databases and methodology, one consistent finding with these studies is the overall increase in the odds of infection-related complications among IBD DM patients compared to the IBD cohorts. This was consistent with our study that found an increase in the odds of having sepsis and septicemia among IBD DM patients.

Several mechanisms might explain the reason for the increased incidence of infectious complications among the IBD DM cohort. IBD and DM are both autoimmune disease conditions that disrupt the entire immune system [16-17]. These disease states are associated with dysregulation of the intestinal immune barrier, promoting local and systemic inflammation, explaining the increased susceptibility to several infections [1819]. The dysregulation also leads to the release of multiple cytokines, including tumor necrosis factor (TNF)alpha and interleukins [20-21]. The inhibited secretions of the interleukins in diabetic patients cause a defect in the antigen-presenting cells, monocytes, and contribute to reduced immunity [22]. Furthermore, the hyperglycemic state in DM affects the complement system and contributes to reduced neutrophil function [23]. Also, IBD patients are usually on immunosuppressive medications, which reduces the function of the overall immune system and predisposes them to an increased risk of opportunistic infections [24].

Given the overlapping impairment in the immune system, concomitant DM is a significant comorbidity among patients with IBD, as reflected by increased hospitalizations [15]. While our study did not find a significant difference in the length of hospital stay and cost of hospitalization between IBD DM and IBD, we did not explore health care utilization. A study found that IBD DM patients had a higher outpatient prescription and antibiotic usage than IBD cohorts [15]. Also, IBD DM patients' increased emergency department and gastrointestinal clinic visits reflected the important burden of IBD DM comorbidity [15].

The use of propensity score matching (PSM) is one of the strengths of this study. The PSM methodology has been compared to the randomized clinical trials, as it effectively adjusts for confounders and produces estimates close to those derived from randomized clinical trials [25-26]. PSM facilitates comparability between the IBD DM and IBD cohorts, making it a valuable technique for assessing the risk factors between these groups. Therefore, PSM minimizes several biases and limitations of a large observational study like ours. The additional strengths of our study include utilizing an extensive, nationwide inpatient database, which makes the results generalizable to the entire population. Despite these strengths, our findings still needed to be interpreted in light of some limitations. The comorbidities in the nationwide inpatient sample database rely heavily on using the diagnostic coding ICD-10. Any error in coding can affect the study's validity. Thus, there might be variations, which is also a limitation that could affect the results generated. The PSM methodology was designed to minimize confounding that could bias the results from observed covariates. However, PSM cannot address unobserved factors, which may still lead to biased results. Also, our study did not explore the different outcomes regarding the variants of IBD. IBD, comprising Crohn's disease and ulcerative colitis, can have different results. The retrospective nature of this study is a limitation and the diagnosis of diabetes mellitus based on ICD-10 codes alone might be unreliable, thus some of the patients in the IBD-only group might also have diabetes mellitus but are yet to be diagnosed, which can affect the results of this study. A prospective study will be helpful to better study the complications of inflammatory bowel disease in patients with diabetes mellitus.

\section{Conclusions}

This study shows that DM is a determinant of severe disease and increased risk of severe infectious complications in hospitalized diabetic patients with IBD. The use of PSM is one of the strengths of this study, as it effectively adjusts for confounders and minimizes several biases and limitations of a large observational study. More longitudinal and prospective studies are required to further study the impact of diabetes among patients with IBD.

\section{Additional Information}




\section{Disclosures}

Human subjects: Consent was obtained or waived by all participants in this study. Animal subjects: All authors have confirmed that this study did not involve animal subjects or tissue. Conflicts of interest: In compliance with the ICMJE uniform disclosure form, all authors declare the following: Payment/services info: All authors have declared that no financial support was received from any organization for the submitted work. Financial relationships: All authors have declared that they have no financial relationships at present or within the previous three years with any organizations that might have an interest in the submitted work. Other relationships: All authors have declared that there are no other relationships or activities that could appear to have influenced the submitted work.

\section{References}

1. Hatoum OA, Binion DG: The vasculature and inflammatory bowel disease: contribution to pathogenesis and clinical pathology. Inflamm Bowel Dis. 2005, 11:304-13. 10.1097/01.mib.0000160772.78951.61

2. Ghosh S, Mitchell R: Impact of inflammatory bowel disease on quality of life: results of the European Federation of Crohn's and Ulcerative Colitis Associations (EFCCA) patient survey. J Crohns Colitis. 2007, 1:10-20. 10.1016/j.crohns.2007.06.005

3. Vidal A, Gómez-Gil E, Sans M, Portella MJ, Salamero M, Piqué JM, Panés J: Health-related quality of life in inflammatory bowel disease patients: the role of psychopathology and personality. Inflamm Bowel Dis. 2008, 14:977-83. 10.1002/ibd.20388

4. Alatab S, Sepanlou SG, Ikuta K, et al.: The global, regional, and national burden of inflammatory bowel disease in 195 countries and territories, 1990-2017: a systematic analysis for the Global Burden of Disease Study 2017. Lancet Gastroenterol Hepatol. 2020, 5:17-30. 10.1016/S2468-1253(19)30333-4

5. Kim DH, Cheon JH: Pathogenesis of inflammatory bowel disease and recent advances in biologic therapies . Immune Netw. 2017, 17:25-40. 10.4110/in.2017.17.1.25

6. $\mathrm{Ng} \mathrm{SC}$, Bernstein CN, Vatn MH, et al.: Geographical variability and environmental risk factors in inflammatory bowel disease. Gut. 2013, 62:630-49. 10.1136/gutjnl-2012-303661

7. Sinh P, Tabibian JH, Biyani PS, Mehta K, Mansoor E, Loftus EV Jr, Dave M: Inflammatory bowel disease does not impact mortality but increases length of hospitalization in patients with acute myocardial infarction. Dig Dis Sci. 2021, [Epub ahead of print]:10.1007/s10620-020-06818-X

8. Rothfuss KS, Stange EF, Herrlinger KR: Extraintestinal manifestations and complications in inflammatory bowel diseases. World J Gastroenterol. 2006, 12:4819-31. 10.3748/wjg.v12.i30.4819

9. Afzali A, Katz S: Inflammatory bowel disease in the baby to baby boomer: pediatric and elderly onset of IBD . Curr Treat Options Gastroenterol. 2018, 16:289-305. 10.1007/s11938-018-0188-9

10. Kumar A, Teslova T, Taub E, Miller JD, Lukin DJ: Comorbid diabetes in inflammatory bowel disease predicts adverse disease-related outcomes and infectious complications. Dig Dis Sci. 2021, 66:2005-13. 10.1007/s10620-020-06439-4

11. Kharroubi AT, Darwish HM: Diabetes mellitus: the epidemic of the century . World J Diabetes. 2015, 6:85067. 10.4239/wjd.v6.i6.850

12. Centers for Disease Control and Prevention. National Diabetes Statistics Report, 2020. Estimates of diabetes and its burden in the United States. (2020). Accessed: May 4, 2021: https://www.cdc.gov/diabetes/data/statistics-report/index.html.

13. Choi JK, Kim DW, Shin SY, Park EC, Kang JG: Effect of ulcerative colitis on incidence of colorectal cancer: results from the Nationwide Population-Based Cohort Study (2003-2013). J Cancer. 2016, 7:681-6. 10.7150/jca.14264

14. Fitzmorris PS, Colantonio LD, Torrazza Perez E, Smith I, Kakati DD, Malik TA: Impact of metabolic syndrome on the hospitalization rate of Crohn's disease patients seen at a tertiary care center: a retrospective cohort study. Digestion. 2015, 91:257-62. 10.1159/000380763

15. Din H, Anderson AJ, Ramos Rivers C, et al.: Disease characteristics and severity in patients with inflammatory bowel disease with coexistent diabetes mellitus. Inflamm Bowel Dis. 2020, 26:1436-42. 10.1093/ibd/izz305

16. Calle MC, Fernandez ML: Inflammation and type 2 diabetes. Diabetes Metab. 2012, 38:183-91. 10.1016/j.diabet.2011.11.006

17. Jurjus A, Eid A, Al Kattar S, et al.: Inflammatory bowel disease, colorectal cancer and type 2 diabetes mellitus: the links. BBA Clin. 2016, 5:16-24. 10.1016/j.bbacli.2015.11.002

18. Nishida A, Inoue R, Inatomi O, Bamba S, Naito Y, Andoh A: Gut microbiota in the pathogenesis of inflammatory bowel disease. Clin J Gastroenterol. 2018, 11:1-10. 10.1007/s12328-017-0813-5

19. Zuo T, Ng SC: The gut microbiota in the pathogenesis and therapeutics of inflammatory bowel disease . Front Microbiol. 2018, 9:2247. 10.3389/fmicb.2018.02247

20. Knudsen SH, Pedersen BK: Targeting inflammation through a physical active lifestyle and pharmaceuticals for the treatment of type 2 diabetes. Curr Diab Rep. 2015, 15:82. 10.1007/s11892-015-0642-1

21. Karstoft K, Pedersen BK: Exercise and type 2 diabetes: focus on metabolism and inflammation . Immunol Cell Biol. 2016, 94:146-50. 10.1038/icb.2015.101

22. Casqueiro J, Casqueiro J, Alves C: Infections in patients with diabetes mellitus: a review of pathogenesis . Indian J Endocrinol Metab. 2012, 16 Suppl 1:S27-36. 10.4103/2230-8210.94253

23. Stegenga ME, van der Crabben SN, Blümer RM, et al.: Hyperglycemia enhances coagulation and reduces neutrophil degranulation, whereas hyperinsulinemia inhibits fibrinolysis during human endotoxemia. Blood. 2008, 112:82-9. 10.1182/blood-2007-11-121723

24. Shah ED, Farida JP, Siegel CA, Chong K, Melmed GY: Risk for overall infection with anti-TNF and antiintegrin agents used in IBD: a systematic review and meta-analysis. Inflamm Bowel Dis. 2017, 23:570-7. 10.1097/MIB.0000000000001049

25. Okoli GN, Sanders RD, Myles P: Demystifying propensity scores. Br J Anaesth. 2014, 112:13-5. 10.1093/bja/aet290 


\section{Cureus}

26. Littnerova S, Jarkovsky J, Parenica J, Pavlik T, Spinar J, Dusek L: Why to use propensity score in observational studies? Case study based on data from the Czech clinical database AHEAD 2006-09 [Article in Czech]. Cor et Vasa. 2013, 55: e383-90. 10.1016/j.crvasa.2013.04.001 